Umweltorientiertes Kostenmanagement in China

\title{
Erste Erfolge für Firmen und Umwelt
}

\begin{abstract}
Mit Gesetzen zur umweltgerechten Produktion versucht China die industrielle Umweltbelastung zu verringern. Erfahrungen aus der Boom-Provinz Zhejiang zeigen, wie Unternehmen auf die neuen Vorschriften reagieren und welche Herausforderungen trotz erster Erfolge bleiben. Von Susanne Arlinghaus
\end{abstract}

$F^{2}$ st täglich berichten Radio, Fernsehen und Zeitungen über die entwürdigenden Arbeitsbedingungen in chinesischen Fabriken und die katastrophalen Auswirkungen industrieller Produktion auf die Umwelt. Die verursachten Schäden werden mittlerweile weltweit spürbar und es beschäftigen sich nicht nur eingeweihte Kreise mit den Problemen des unkontrollierten Wirtschafswachstums in China. Auch im Land selbst nehmen Politiker auf Landes- und Provinzebene Umweltprobleme wahr.

Die globalen Kosten für die massive Zerstörung der Umwelt durch die Verschmutzung von Luft und Wasser sind zwar noch nicht abzusehen, allerdings kann man sichergehen, dass sie ausgesprochen hoch sein werden. Sogar der chinesische Vizeminister für Umwelt Pan Yue hat den viel zu hohen Einsatz von Rohstoffen im Verhältnis zum erreichten Ergebnis öffentlich eingestanden: „Für Produktion von Waren im Wert von 10.000 Dollar“, sagte er in einem Presseinterview, „benötigen wir gut siebenmal so viele Ressourcen wie Japan, fast sechsmal so viele wie die USA und, besonders peinlich, fast dreimal so viele wie Indien“ (Spiegel 2005).

\section{Wie wirkt die Gesetzgebung ...}

Wie das Zitat des Vizeministers andeutet, hat die chinesische Regierung den Ressourcenverbrauch zum Thema gemacht. Die nationale Gesetzgebung zur
Verminderung des Ressourcenverbrauchs entspricht in China international oft gutem Standard oder ist sogar vorbildlich. Beispiele dafür sind das Renewable Energy Law, das im Februar dieses Jahres in Kraft tritt, oder die Regelung des Maximalbenzinverbrauchs von Neuwagen (Oberndörfer 2005). Allerdings folgen den gut gemeinten Gesetzen oftmals keine ausreichenden staatlichen Anstrengungen zur Umsetzung.

Mangelnder staatlicher Nachdruck zeigt sich auch bei der Umsetzung des Cleaner Production (CP) Promotion Law von 2002, das mit dem ersten Januar 2003 wirksam wurde. Das Gesetz soll umweltgerechte Produktionsmethoden in Industriebetrieben verbreiten, um den Ressourcenverbrauch zu verringern, effizienter zu gestalten und umweltschädliche Stoffe zu substituieren (Chen 2005). Allerdings hat dieses Gesetz keine unmittelbar bindende Wirkung für alle Unternehmen (1).

\section{... auf Provinzebene ...}

Die Provinz Zhejiang südlich von Shanghai bemüht sich um das Image einer ökologischen Vorreiterprovinz mit dem Slogan Green Zhejiang. Sie setzt sich für so hohe Ziele wie die Kreislaufwirtschaft ein und fördert in diesem Zusammenhang gezielt die Umsetzung des CP Promotion Law (EECZ 2005). Wie aber kann man Unternehmen an Ressourceneinsparungen heranführen, wenn die dazugehörige Gesetzgebung nicht bindend ist?
Die Provinzregierung von Zhejiang, speziell die Economic and Trade Commission (ETC) und das Environmental Protection Bureau (EPB), werden dazu zwischen 2003 und 2007500 Pilotbetriebe auswählen. Ausgewählte Firmen müssen ein so genanntes Cleaner Production Audit (CP Audit) absolvieren. Die durchführenden Berater werden in einem einwöchigen Kurs vorbereitet. Es liegt aber nahe, dass innerhalb eines solch kurzen CP Audit-Kurses nicht alle Möglichkeiten zur optimalen Nutzung von Ressourcen angesprochen und vermittelt werden.

Gefördert durch die Gesellschaft für technische Zusammenarbeit (GTZ) begannen ETC und EPB in Jahre 2004 parallel zum CP Audit ein weiteres Instrument zu testen, das eine größere Ausschöpfung der Optimierungsmöglichkeiten versprach: Umweltorientiertes Kostenmanagement (UoKM) aus dem Programm Profitables Umweltmanagement (PREMA) (2). Der Ansatz basiert auf dem aus dem Flusskostenmanagement bekannten Non-Product-Output(NPO)-Ansatz und verbindet ihn mit Elementen des Veränderungsmanagements.

Im Falle einer Anwendung gewinnen die Unternehmen dreifach. Der Fokus liegt auf Kosteneinsparungen, die durch optimierte Ressourcennutzung erzielt werden. Zweitens wird die Umweltperformance verbessert. Die Realisierung dieser Potenziale im Unternehmen hängt aber von den Managementmethoden ab. Der dritte Nutzen liegt daher im gezielten Veränderungsmanagement in den Unternehmen, das auch langfristig für die Firmen in den genannten Bereichen zu Vorteilen führt. Erst wenn Kosteneinsparungen und nicht die bloße Erfüllung von Gesetzen oder das Herz für die Umwelt im Vordergrund stehen, kann man davon ausgehen, dass Unternehmen sich von allein bewegen.

\section{... um messbare Resultate zu erreichen ...}

Die sieben Pilotfirmen der UoKM-Einführung zwischen Mai und November 2004 kommen aus so umweltschädlichen Sektoren wie Galvanisierung, der Textil-, 
Pharma- oder chemischen Industrie. Die ersten Resultate können sich sehen lassen. Es sind Maßnahmen entwickelt worden, die insgesamt circa fünf Prozent der jährlichen Produktionskosten einsparen. Diese Einsparungen resultierten aus der Verringerung des Ressourcenverbrauchs, sodass zum Beispiel mehr als $100.000 \mathrm{Li}$ ter Abwasser und etwa 4.700 Tonnen Abfälle jährlich weniger entstehen.

Ein konkreter Fall ist die Firma Lan Tian, ein Produzent von Substituten von Chlorfluorkohlenwasserstoffen. Mit 25 umgesetzten Einzelmaßnahmen sparte sie circa eine Millionen RMB, das heißt drei Prozent ihrer gesamten Produktionskosten ein. Laut Liu Jianpeng, Manager der Produktion bei Lan Tian, ist das Hilfreichste des Programms allerdings die zyklische Methode zur Lösung von Problemen. Diese hat Lan Tian daher auch an weiteren Produktionslinien eingeführt und damit im Jahre 2005 knapp eine weitere halbe Million RMB eingespart (Chen 2005a). Die Pharmafirma Xianju Charioteer verringerte allein circa ein Drittel des Wasserverbrauchs und 20 Prozent des Energieverbrauchs mit den Maßnahmen, die sie im ersten Jahr des Programms entwickelt hatten.

Eine Studie von November 2005 zeigt, dass die Firmen seither UoKM in den Unternehmen verankern (Chen 2005a). Die weitere Nutzung des Instrumentariums wird durch die eingeführten Managementveränderungen gesichert. Beispielsweise wurden in den Firmen Vorschlags- und Bonussysteme für alle Mitarbeiter eingeführt. Ein Mitarbeiter des Unternehmens Xianju Charioteer erhielt zehn bis 15 Prozent der durch die von $i h m$ vorgeschlagene Maßnahme eingesparten Summe als Prämie, ausgezahlt in Form eines Volkswagen Santana.

\section{... ohne dass eine Beratungs- kultur existiert?}

Notwendige Bedingungen, um diese Resultate in den Firmen zu erzielen, sind ein Gesetz, dokumentierter Umsetzungswillen seitens der (Provinz-)Regierung gepaart mit ökonomischem Druck auf die Unternehmen und nicht zuletzt Berater, die einen solchen Prozess adäquat begleiten können.

Dem großen Unternehmensmarkt Chinas steht aber ein noch kaum ausgebildeter Beratermarkt gegenüber. So war es auch Ziel der GTZ, die Ausbildung von Beratern und somit die lokale Verbreitung des Instrumentariums zu fördern. Elf Berater aus neun unterschiedlichen Beratungsfirmen wurden in der ersten Runde im Jahre 2004 an die Beratung herangeführt. Mit dem großen Erfolg bei den Unternehmen wurde eine zweite Runde aufgelegt, in der die Berater der ersten Runde seit Juli 2005 mit weiteren Firmen das Training und die Beratung für die Firmen selbst gestalten. Eine dritte Anwendung ist für 2006 in Planung. In jeder weiteren Runde werden mehr Trainer und Berater an weiteren Standorten ausgebildet, um eine tragfähige Beraterbasis für das Instrument zu erzielen.

\section{Fazit}

Ohne Zweifel hat Umweltorientiertes Kostenmanagement (UoKM) in den Firmen in China gute Erfolge erzielt. Es entpuppt sich als profitabler Weg, die Anforderungen des Cleaner Production Gesetzes zu erfüllen. Vertreter des ETC Zhejiang haben ihre Zufriedenheit mit dem Modell-Programm und den im Vergleich zum CP Audit-Ansatz besseren Resultaten öffentlich bekundet. Allerdings ist es auch ein aufwändiger Weg. Für die beteiligten Firmen wie auch für die Umwelt hat er sich gelohnt.

Aber was sind ein Dutzend Firmen, die bisher an dem Programm teilgenommen haben, was sind gar die wenigen Hundert Firmen auf der Liste der Provinzregierung, gegen zehn Millionen kleine und mittlere Betriebe Chinas? Wie schafft man es in einem so großen, unübersichtlichen Staat mit wenig Tradition in der firmenbezogenen Umweltgesetzgebung, die Masse der Unternehmen zu erreichen?

Angesichts des Ausmaßes und der Vielgestaltigkeit der relevanten Probleme fällt eine einfache Antwort auf die Fragen schwer. Die Entwicklung der Umweltberatungs- und Trainingskompetenzen so- wie auch die Nachfrage nach Beratung seitens der Unternehmen hält mit dem beschleunigten Wirtschaftswachstum noch nicht Schritt. Diese Entwicklungen bedürfen weiterhin der Unterstützung durch den Staat. Insofern ist Zhejiang mit seinen Vorstößen sicherlich auf einem guten Weg. So wie Zhejiang einen Wettbewerb um die besten Erfolge auf Firmenebene erwirkt, nehmen auch andere chinesischen Provinzen die Herausforderung an: bereits acht haben ein Eco-Province-Programm aufgelegt. Es bleibt zu hoffen, dass sich die Vorreiterrolle Zhejiang noch weiter entwickelt.

\section{Literatur \\ Chen, J.: How can Environment-oriented Cost Management (EoCM) contribute to the imple- mentation of the CP law in Zhejiang in China? Unpublished Master Thesis. Freiberg 2005. \\ Chen, J.: Sustainability Monitoring of the EoCM pro- gramme in Zhejiang 2004. Hangzhou 2005a. \\ Environment-oriented Enterprise Consultancy Zhejiang (EECZ) (Ed.): How to increase profits with Cleaner Production? Hangzhou 2005. \\ Oberndörfer, U.: Umwelt- und Energiestrategien in China - Erneuerbare Energien ohne energische Nachhaltigkeitspolitik. In: Ökologisches Wirtschaften 4/2005. \\ Spiegel: Angst vor dem Crash. In: Spiegel Special 7/ 2005, S.76}

\section{Anmerkungen \\ (1) Siehe Artikel 28 i.V.m. Artikel 40 unter www.sinoitaenvironment.org/ ReadNewsxi.asp?News|D=2028 \\ (2) Siehe www.premanet.net}

AUTORIN + KONTAKT Susanne Arlinghaus ist freie Beraterin und Trainerin für Umweltmanagement und Organisationsentwicklung in Köln. Sie war als Trainerin an der Einführung von UoKM in Zhejiang beteiligt.

E-Mail: Mail@Arlinghaus.info 
(c) 20I0 Authors; licensee IÖW and oekom verlag. This is an article distributed under the terms of the Creative Commons Attribution Non-Commercial No Derivates License (http://creativecommons.org/licenses/by-nc-nd/3.o/), which permits unrestricted use, distribution, and reproduction in any medium, provided the original work is properly cited. 